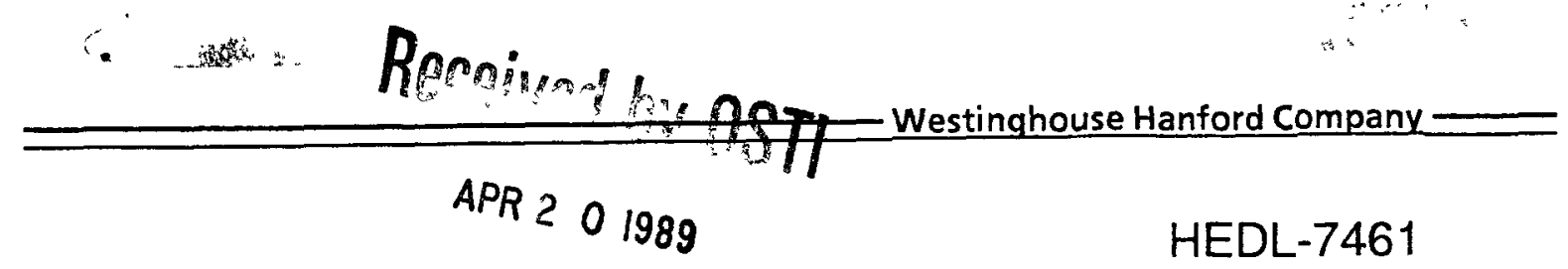

\title{
Summary of the EBR-II AD-2 Ferritics Irradiation Experiment
}

HEDL --7461

DE89 010141

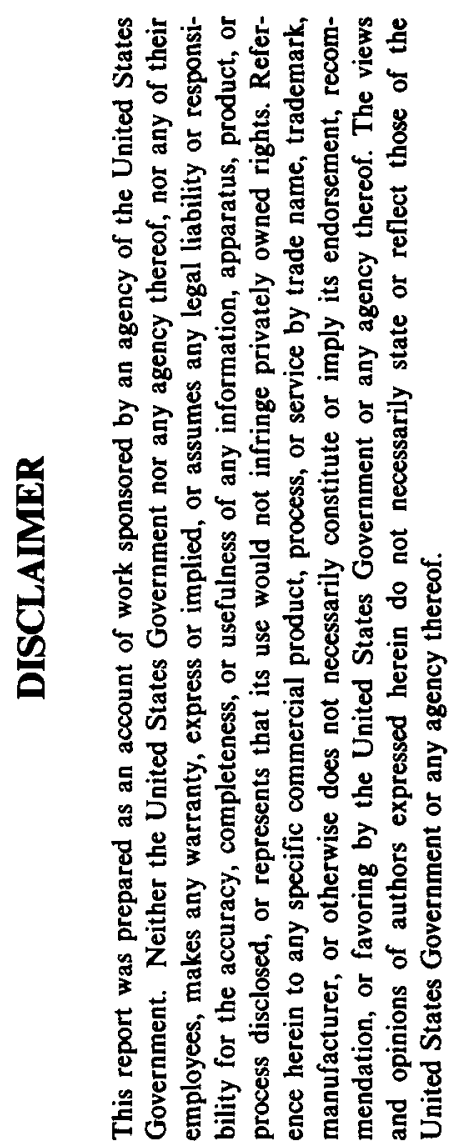

A. M. Ermi

June 1984

Prepared for the U.S. Department of Energy Assistant Secretary for Fusion Energy

HANFORD ENGINEERING DEVELOPMENT LABORATORY - Operated by Westinghouse Hanford Company, P.O. BOX 1970, Richland, WA, a Subsidrary of Westinghouse Electric Corporation, under Contract No. DE-AC06-76FF02170. 


\section{DISCLAIMER}

Portions of this document may be illegible in electronic image products. Images are produced from the best available original document. 


\section{NOTICE}

This report was prepared as an account of work sponsored by the United States Government. Neither the United States nor the U.S. Department of Energy (DOE), nor any of its employes, nor any of its contractors, subcontractors or their employes, makes any warranty, expressed or implied, or assumes any legal liability or responsibility for any third party's use or the results of such use of any information, apparatus, product or process disclosed in this report, or represents that its use by such third party would not infringe privately owned rights. 
2.4 SUMMARY OF THE EBR-II AD-2 FERRITICS IRRADIATION EXPERIMENT - A. M. Ermi

(Westinghouse Hanford Company)

\subsubsection{ADIP Task}

ADIP Program Task: Ferritic Stee 1s Development (Path E).

\subsubsection{Dbjectives}

The objective of the AD-2 experiment is to provide baseline, high fluence data at several temperatures for the ferritic alloys HT-9 and $9 \mathrm{Cr}-1$ Mo. Developmental $2-1 / 4 \mathrm{Cr}-1$ Mo is also being scoped in this experiment. The AD-2 experiment is designed to yield data concerning the irradiation effects on the mechanical properties of these al loys with emphas is on their tensile, fatigue, fracture toughness and weld properties. Characterization of the irradiation effects on the microstructure of these alloys will also be performed.

\subsubsection{Summary}

Six uninstrumented B-7C capsules were removed from the EBR-II after undergoing irradiation during Cycies 109-113 (August 1980 to June 1981). Several specimens were removed and distributed for interim examination, while the remaining specimens were re-encapsulated into four new $B-7 \mathrm{C}$ capsules. In addition, some unirradiated specimens were included in the new capsules. These capsules were removed after undergoing irradiation during Cycles 118-121 and 123 (February 1982 to April 1983).

\subsubsection{Progress and Status}

\subsubsection{Introduction}

The AD-2 experiment has been designed to provide mechanical properties data on selected ferritic alloys for application in fusion reactor first walls and blankets. of particular concern are the irradiation effects on the fracture and weld properties of the materials. Alloy selection and irradiation temperatures have been specified by the Alloy Development for Near Term Applications Working Group. This is the final report on the AD-2 experiment, which summarizes the experimental parameters for both the initial and reconstitution test matrices.

\subsubsection{Test Matrices}

Details regarding specimen preparation, heat treatments, test matrices and capsule loadings have been reported in previqus ADIP Progress Reports. The initial specimen preparation and igading were reported in June 1980 , while the reconstitution test matrix was reported in March 1982.2,3 The complete $\mathrm{AD}-2$ test matrix encompassing both irradiations is detailed in Table 2.4.1.

\subsubsection{Irradiation Parameters}

The initial irradiation utilized six uninstrumented $\mathrm{B}-7 \mathrm{C}$ capsules, two at both $390^{\circ} \mathrm{C}$ and $500^{\circ} \mathrm{C}$, and one at each $450^{\circ} \mathrm{C}$ and $550^{\circ} \mathrm{C}$. These capsules were inserted into the EBR-II in August 1980 for Cycle 109 and were removed in June 1981 after Cycle 113 . Specimens in the peak flux position accumulated a fluence of $13.5 \mathrm{dpa}$ (based on $4.8 \mathrm{dpa} / 10^{22} \mathrm{n} / \mathrm{cm}^{2}$ for $\mathrm{HT}-9$ ).

The second irradiation, including reconstituted specimens plus new, unirradiated specimens, utilized four B-7C capsules, one at each of the four temperatures. These capsules were inserted in February 1982 for Cycle 118 and were removed in April 1983 after Cycle 123. (The capsules were removed during the transient cycle, 122). Specimens in the peak flux position during the second irradiation accumulated a fluence of 16.2 dpa. Reconstituted specimens in the peak positions for both irradiations accumulated $29.7 \mathrm{dpa}$.

A summary of the experimental parameters for the entire AD-2 experiment is given in Table 2.4.2. Included are the fluences for each irradiation as a function of the seven levels along the $B-7 \mathrm{C}$ capsules. Details of fluences for particular specimens are available from the author on request.

Specimens from the AD-2 experiment were distributed as follows: ORNL received all tensile, Grodzinski fatigue and fatigue initiation specimens; WHC received all fracture toughness and fatigue crack growth specimens; the Charpy specimens were distributed to both ORNL and WHC; and the TEM disk Specimens were distributed to various participants in the ADIP and DAFS programs.

\subsubsection{Future Work}

This concludes the irradiation phase of the $A D-2$ experiment. 


\subsubsection{References}

1. R. J. Puigh and N. F. Panayotou, "Specimen Preparation and Loading for the AD-2 Ferritics Experiment", pp. 260-293 in ADIP Semiannu. Prog. Rep. June 1980, DOE/ER-0045-3, U.S. DOE, Office of Fusion Energy.

2. D. T. Peterson, "TEM Specimen Matrix for the AD-2 Reconstitution", pp. 13-16 ADIP Semiannu. Prog. Rep. March 1982, DOE/ER-0045/8, U.S. DOE, Office of Fusion Energy.

3. A. M. Ermi, "Reconstitution of the AD-2 Ferritics Experiment", pp. 431-441 in ADIP Semiannu. Prog. Rep. March 1982, DOE/ER-0045/8, U.S. DOE, Office of Fusion Energy. 
Table 2.4.1. AD-2 Test Matrix

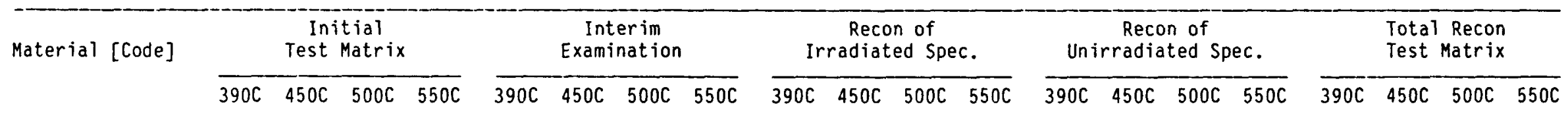

IENSILE [T]

\begin{tabular}{|c|c|c|c|c|c|c|c|c|c|c|c|c|c|c|c|c|c|c|c|c|}
\hline$H T-9(\# 1)[U H]$ & 20 & 10 & 20 & 10 & 10 & 5 & 10 & 5 & 10 & 5 & 10 & 5 & -- & -- & - & -- & 10 & 5 & 10 & 5 \\
\hline$H T-9(\# 2)[T W]$ & 10 & 5 & 10 & 5 & 10 & 2 & 10 & 2 & -- & 3 & - & 3 & -- & -- & -- & -- & -- & 3 & -- & 3 \\
\hline$H T-9(X A A-3587)[S B]$ & -- & -- & -- & -- & -- & -- & -- & -- & $=-$ & -- & -- & -- & 4 & 4 & 1 & 4 & 4 & 4 & 1 & 4 \\
\hline$H T-9,1 N i[S C]$ & $\cdots$ & -- & -- & - & -- & -- & -- & -- & -- & -- & -- & -- & 4 & 4 & -- & 2 & 4 & 4 & - & 2 \\
\hline $\mathrm{HT}-9,2 \mathrm{Ni}[\mathrm{SD}]$ & -- & -- & -- & -- & - & -- & -- & -- & - & -- & - & - & 4 & 4 & 1 & 4 & 4 & 4 & 1 & 4 \\
\hline $\mathrm{HT}-9,2 \mathrm{Ni}$ Adj $[\mathrm{SE}]$ & -- & - & -- & -- & -- & -- & -- & -- & -- & -- & -- & - & 4 & -- & -- & -- & 4 & -- & - & -- \\
\hline $9 \mathrm{Cr}-1 \mathrm{Mo}(30182)[\mathrm{XT}]$ & 9 & 3 & 9 & 6 & 4 & 1 & 4 & 3 & 5 & 2 & 5 & 3 & -- & - & -- & -- & 5 & 2 & 5 & 3 \\
\hline $9 \mathrm{Cr}-1 \mathrm{Mo}(\mathrm{XA}-3590)[\mathrm{TA}]$ & ]-- & -- & -- & - & -- & -- & -- & - & -- & -- & -- & $\cdots$ & 4 & 4 & -- & 4 & 4 & 4 & -- & 4 \\
\hline $9 \mathrm{Cr}-1 \mathrm{Mo}-2 \mathrm{Ni}[\mathrm{TB}]$ & -- & -- & $=-$ & -- & -- & -- & -- & -- & - & -- & - & - & 4 & 4 & 1 & 4 & 4 & 4 & 1 & 4 \\
\hline $9 \mathrm{Cr}-1 \mathrm{MO}-2 \mathrm{Ni}$ Adj $[\mathrm{TC}]$ & -- & - & -- & -- & -- & -- & -- & -- & -- & -- & -- & -- & - & 4 & $-\infty$ & -- & -- & 4 & - & - \\
\hline $2-1 / 4 \mathrm{Cr}-1 \mathrm{Mo}[Z \mathrm{ZT}]$ & 6 & 3 & 6 & 3 & 3 & 1 & 3 & 1 & 3 & 2 & 3 & 2 & -- & -- & - & -- & 3 & 2 & 3 & 2 \\
\hline $\operatorname{LRO}-160[6]$ & -- & - & -- & -- & -- & -- & $=-$ & -- & -- & -- & -- & -- & -- & - & -- & 2 & -- & - & -- & 2 \\
\hline LRO-42 [VH] & -- & - & -- & -- & -- & -- & -- & -- & -- & -- & -- & -- & 2 & 2 & -- & 2 & 2 & 2 & - & 2 \\
\hline
\end{tabular}

CRACK GROWTH [CG]

\begin{tabular}{|c|c|c|c|c|c|c|c|c|c|c|c|c|c|c|c|c|c|c|c|c|}
\hline $\mathrm{HT}-9(\# 1)[\mathrm{HT}]$ & 22 & 11 & 22 & 11 & 10 & 4 & 13 & 4 & 12 & 7 & 9 & 7 & -- & -- & - & - & 12 & 7 & 9 & 7 \\
\hline$H T-9(\| 2)[M R]$ & 10 & 5 & 10 & 5 & 10 & 2 & 10 & 2 & -- & 3 & -- & 3 & - & -- & -- & -- & -- & 3 & -- & 3 \\
\hline $9 \mathrm{Cr}-1 \mathrm{Mo}(30182)[\mathrm{AS}]$ & 10 & 5 & 10 & 5 & 4 & 2 & 4 & 2 & 6 & 3 & 6 & 3 & -- & -- & - & -- & 6 & 3 & 6 & 3 \\
\hline $2-1 / 4 \mathrm{Cr}-1 \mathrm{Mo}[\mathrm{ZC}]$ & 10 & 5 & 10 & 5 & 4 & 2 & 10 & 2 & 6 & 3 & -- & 3 & -- & -- & -- & -- & 6 & 3 & -- & 3 \\
\hline$H T-9(W)[R W]$ & 10 & -- & 10 & 10 & 4 & -- & 5 & 4 & 6 & -- & 5 & 6 & - & -- & -- & -- & 6 & -- & 5 & b \\
\hline$H T-9(H A \bar{Z})[W R]$ & 5 & -- & 5 & 5 & 2 & $\cdots$ & 2 & 2 & 3 & -- & 3 & 3 & - & -- & -- & -- & 3 & -- & 3 & 3 \\
\hline HT-9(W/HAZ $)[R W]$ & 2 & -- & 2 & 2 & 2 & - & 2 & 2 & -- & -- & -- & -- & -- & -- & -- & -- & -- & -- & $\cdots$ & -1 \\
\hline $9 \mathrm{Cr}-1 \mathrm{Mo}(W)[W M]$ & 6 & -- & 6 & 6 & 2 & -- & 2 & 2 & 4 & -- & 4 & 4 & -- & -- & -- & -- & 4 & -- & 4 & \\
\hline
\end{tabular}

FATIGUE INITIATION [FI]

\begin{tabular}{|c|c|c|c|c|c|c|c|c|c|c|c|c|c|c|c|c|c|c|c|c|}
\hline $\begin{array}{l}H T-9(\| 4)[S F] \\
9 \mathrm{Cr}-1 \mathrm{Mo}(30182)[\mathrm{SI}]\end{array}$ & $\begin{array}{l}6 \\
6\end{array}$ & $\begin{array}{l}3 \\
3\end{array}$ & $\begin{array}{l}6 \\
6\end{array}$ & $\begin{array}{l}3 \\
3\end{array}$ & $\begin{array}{l}4 \\
4\end{array}$ & $\begin{array}{l}3 \\
3\end{array}$ & $\begin{array}{l}4 \\
4\end{array}$ & $\begin{array}{l}3 \\
3\end{array}$ & $\begin{array}{l}2 \\
2\end{array}$ & -- & $\begin{array}{l}2 \\
2\end{array}$ & $\begin{array}{l}-- \\
--\end{array}$ & $\begin{array}{ll}-- \\
--\end{array}$ & $\begin{array}{l}-- \\
--\end{array}$ & $\begin{array}{l}-- \\
--\end{array}$ & -- & $\begin{array}{l}2 \\
2\end{array}$ & $\begin{array}{l}-- \\
--\end{array}$ & $\begin{array}{l}2 \\
2\end{array}$ & $\begin{array}{l}-- \\
--\end{array}$ \\
\hline \multicolumn{21}{|c|}{ GRODZINSK I FATIGUE [GF] } \\
\hline $\begin{array}{l}\mathrm{HT}-9(H 1)[\mathrm{SG}] \\
9 \mathrm{Cr}-1 \mathrm{Mo}(30182)[\mathrm{SH}]\end{array}$ & $\begin{array}{r}10 \\
2\end{array}$ & $\begin{array}{r}5 \\
--\end{array}$ & $\begin{array}{r}10 \\
2\end{array}$ & $\begin{array}{l}5 \\
2\end{array}$ & $\begin{array}{r}10 \\
2\end{array}$ & $\begin{array}{r}3 \\
--\end{array}$ & $\begin{array}{r}10 \\
2\end{array}$ & $\begin{array}{l}3 \\
2\end{array}$ & $\begin{array}{l}-- \\
--\end{array}$ & 2 & -- & $\begin{array}{r}2 \\
--\end{array}$ & -- & $\begin{array}{l}-- \\
--\end{array}$ & $\begin{array}{l}-- \\
--\end{array}$ & $\begin{array}{l}-- \\
--\end{array}$ & -- & $\begin{array}{r}2 \\
--\end{array}$ & -- & $\begin{array}{r}2 \\
--\end{array}$ \\
\hline
\end{tabular}

continued 
Table 2.4.1. AD-2 Test Matrix (cont'd)

\begin{tabular}{|c|c|c|c|c|c|c|c|c|c|c|c|c|c|c|c|c|c|c|c|}
\hline \multirow[t]{2}{*}{ Material [Code] } & \multicolumn{4}{|c|}{$\begin{array}{l}\text { Initial } \\
\text { Test Matrix }\end{array}$} & \multicolumn{4}{|c|}{$\begin{array}{c}\text { Interim } \\
\text { Examination }\end{array}$} & \multicolumn{4}{|c|}{$\begin{array}{c}\text { Recon of } \\
\text { Irradiated Spec. }\end{array}$} & \multicolumn{4}{|c|}{$\begin{array}{l}\text { Recon of } \\
\text { Unirradiated Spec. }\end{array}$} & \multicolumn{3}{|c|}{$\begin{array}{l}\text { Total Recon } \\
\text { Test Matrix }\end{array}$} \\
\hline & $390 \mathrm{C}$ & $450 \mathrm{C}$ & $500 \mathrm{C}$ & $550 \mathrm{C}$ & $390 \mathrm{C}$ & $450 \mathrm{C}$ & $500 \mathrm{C}$ & $550 \mathrm{C}$ & $390 \mathrm{C}$ & $450 \mathrm{C}$ & $500 \mathrm{C}$ & $550 \mathrm{C}$ & $390 \mathrm{C}$ & $450 \mathrm{C}$ & $500 \mathrm{C}$ & $550 \mathrm{C}$ & $390 \mathrm{C}$ & $450 \mathrm{C}$ & $550 \mathrm{C}$ \\
\hline
\end{tabular}

FRACTURE TOUGHNESS [FT]

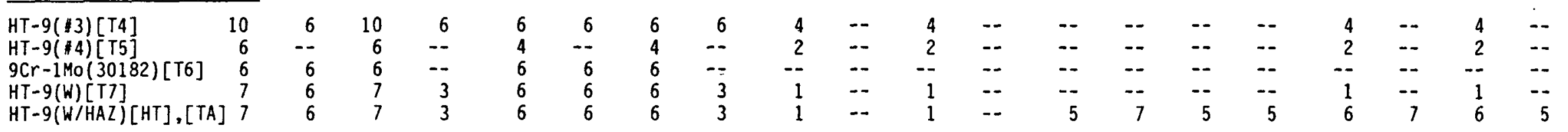

HT-9(W/HAZ) [HT], [TA] 7

$\frac{\text { CHARPY [C] }}{\text { HT-9(I1)[KT] }}$

$H T-9(11)[K T]$
$H T-9(13)[T T]$

HT-9(XAA-3587)[SB]

$H T-9,1 N i[S C]$

HT $-9,2 \mathrm{Ni}[\mathrm{SD}]$

$9 \mathrm{Cr}-1 \mathrm{Mo}(30176)[\mathrm{AF}]$

$9 \mathrm{Cr}-1 \mathrm{Mo}(30182)$ [TV]

$9 \mathrm{Cr}-1 \mathrm{Mo}(\mathrm{XA}-3590)$ [TA] -

$9 \mathrm{Cr}-1 \mathrm{Mo}-2 \mathrm{Ni}[\mathrm{TB}]$

$\mathrm{HT}-9(\mathrm{~W})[\mathrm{Tl}]$

HT-g(W/HAZ) [TX]

$9 \mathrm{Cr}-\mathrm{IMO}(\mathrm{W})[\mathrm{NL}]$

DISK PACKETS [D]

Various Alloys

$\begin{array}{rrrrrrrrrrrrrrrrrrrrrrr}-- & -- & -- & -- & -- & -- & -- & -- & -- & -- & -- & -- & 8 & 8 & -- & 8 & 8 & 8 & -- & 8 \\ 10 & 8 & 10 & 8 & 6 & 4 & 6 & 4 & 4 & 4 & 4 & 4 & -- & -- & -- & -- & 4 & 4 & 4 & 4 \\ -- & -- & -- & -- & -- & -- & -- & -- & -- & -- & -- & -- & 5 & -- & -- & -- & 5 & -- & -- & -- \\ -- & -- & -- & -- & -- & -- & -- & -- & -- & -- & -- & -- & -- & -- & 3 & -- & -- & -- & 3 & -- \\ -- & -- & -- & -- & -- & -- & -- & -- & -- & -- & -- & -- & 3 & -- & 3 & -- & 3 & -- & 3 & -- \\ -- & -- & -- & -- & -- & -- & -- & -- & -- & -- & -- & -- & 8 & 8 & 6 & 8 & 8 & 8 & 6 & 8 \\ 8 & 4 & 8 & 4 & 4 & 4 & 4 & 4 & 4 & -- & 4 & -- & -- & -- & -- & -- & 4 & -- & 4 & -- \\ -- & -- & -- & -- & -- & -- & -- & -- & -- & -- & -- & -- & -- & 5 & -- & 5 & -- & 5 & -- & 5 \\ -- & -- & -- & -- & -- & -- & -- & -- & -- & -- & -- & -- & -- & 3 & -- & 3 & -- & 3 & -- & 3 \\ 7 & 4 & 7 & 4 & 7 & 4 & 7 & 4 & -- & -- & -- & -- & -- & -- & -- & -- & -- & -- & -- & -- \\ 7 & -- & 7 & -- & 7 & -- & 7 & -- & -- & -- & -- & -- & -- & -- & -- & -- & -- & -- & -- & -- \\ -- & -- & -- & -- & -- & -- & -- & -- & -- & -- & -- & -- & 8 & 8 & 8 & 8 & 8 & 8 & 8 & 8\end{array}$


Table 2.4.2. Summary of the EBR-II AD-2 Experiment

\begin{tabular}{|c|c|c|c|c|}
\hline n & \multicolumn{2}{|c|}{$A D-2$} & \multicolumn{2}{|c|}{$A D-2$ RECON } \\
\hline Date In Reactor: & \multicolumn{2}{|c|}{ August 1980 - June 1981} & \multicolumn{2}{|c|}{ February 1982 - April 1983} \\
\hline Cycle Numbers: & \multicolumn{2}{|c|}{$109-113$} & \multicolumn{2}{|c|}{$118-121 \& 123$} \\
\hline Reactor Power: & \multicolumn{2}{|c|}{$57.25 \mathrm{MW}$} & \multicolumn{2}{|c|}{$57.25 \mathrm{MW}$} \\
\hline Accumulated Exposure: & \multicolumn{2}{|c|}{$10,680 \mathrm{MWD}$} & \multicolumn{2}{|c|}{12,780 MWD } \\
\hline $\begin{array}{l}\text { Capsules/ } \\
\text { Temperature: }\end{array}$ & \multicolumn{2}{|c|}{$\begin{array}{l}\mathrm{B}-317 / 390^{\circ} \mathrm{C} \\
\mathrm{B}-318 / 500^{\circ} \mathrm{C} \\
\mathrm{B}-319 / 550^{\circ} \mathrm{C} \\
\mathrm{B}-320 / 390^{\circ} \mathrm{C} \\
\mathrm{B}-321 / 450^{\circ} \mathrm{C} \\
\mathrm{B}-322 / 500^{\circ} \mathrm{C}\end{array}$} & \multicolumn{2}{|c|}{$\begin{array}{l}\mathrm{B}-317 \mathrm{a} / 390^{\circ} \mathrm{C} \\
\mathrm{B}-318 \mathrm{a} / 450^{\circ} \mathrm{C} \\
\mathrm{B}-319 \mathrm{a} / 500^{\circ} \mathrm{C} \\
\mathrm{B}-321 \mathrm{a} / 550^{\circ} \mathrm{C}\end{array}$} \\
\hline Subassembly: & \multicolumn{2}{|c|}{$X-344 B$} & \multicolumn{2}{|c|}{$x-344 B$} \\
\hline Core Position: & \multicolumn{2}{|c|}{$4 \mathrm{C} 2$} & \multicolumn{2}{|c|}{482} \\
\hline Peak Fluence ${ }^{a}$ : & \multicolumn{2}{|c|}{$\begin{array}{c}2.82 \times 10^{22} \mathrm{n} / \mathrm{cm}^{2} \\
(13.5 \mathrm{dpa})\end{array}$} & \multicolumn{2}{|c|}{$\begin{array}{c}3.37 \times 10^{22} \mathrm{n} / \mathrm{cm}^{2} \\
(16.2 \mathrm{dpa})\end{array}$} \\
\hline $\begin{array}{l}\text { Reactor Level } \\
\text { Number Within } \\
\text { Each Capsule }\end{array}$ & $\begin{array}{l}\text { Distance } \\
\text { From Core } \\
\text { Mid-Plane }\end{array}$ & $\begin{array}{c}\text { Fluence }{ }^{b} \\
10^{22} \mathrm{n} / \mathrm{cm}^{2} \\
(\mathrm{dpa})\end{array}$ & $\begin{array}{l}\text { Distance } \\
\text { From Core } \\
\text { Mid-Plane }\end{array}$ & $\begin{array}{c}\text { Fluence }{ }^{\mathrm{b}} \\
10^{22} \mathrm{n} / \mathrm{cm}^{2} \\
(\mathrm{dpa}) \\
\end{array}$ \\
\hline $\begin{array}{l}1 \\
2 \\
3 \\
4 \\
5 \\
6 \\
7\end{array}$ & $\begin{array}{r}158 \mathrm{~mm} \\
108 \mathrm{~mm} \\
56 \mathrm{~mm} \\
0 \mathrm{~mm} \\
-54 \mathrm{~mm} \\
-102 \mathrm{~mm} \\
-152 \mathrm{~mm}\end{array}$ & $\begin{array}{ll}1.88 & (9.0) \\
2.35 & (11.3) \\
2.67 & (12.8) \\
2.82 & (13.5) \\
2.76 & (13.3) \\
2.53 & (12.1) \\
2.10 & (10.1)\end{array}$ & $\left\{\begin{array}{r}159 \mathrm{~mm} \\
109 \mathrm{~mm} \\
56 \mathrm{~mm} \\
0 \mathrm{~mm} \\
-53 \mathrm{~mm} \\
-102 \mathrm{~mm} \\
-155 \mathrm{~mm}\end{array}\right.$ & $\begin{array}{ll}2.24 & (10.8) \\
2.80 & (13.5) \\
3.20 & (15.4) \\
3.37 & (16.2) \\
3.31 & (15.9) \\
3.02 & (14.5) \\
2.48 & (11.9)\end{array}$ \\
\hline
\end{tabular}

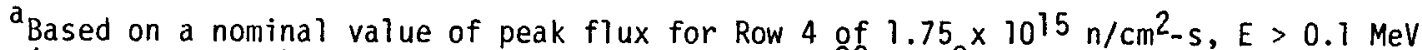
(from dosimetry); dpa values based on $4.8 \mathrm{dpa} / 10^{22} \mathrm{n} / \mathrm{cm}^{2}$ for HT-9 (HEDL TC-160-1, 1974).

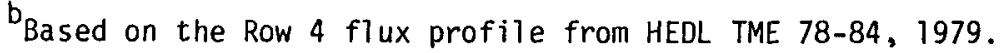

